\title{
Manejo de pastizales en sistemas de producción ganaderos de Nueva Guinea, Costa Caribe Sur de Nicaragua
}

\author{
Carlos Álvarez Amador ${ }^{1}$ \\ Wilberto Antonio Cruz Pastora ${ }^{2}$
}

\section{Resumen}

Esta investigación se encaminó a la caracterización del manejo de las pasturas que hacen los ganaderos de Nueva Guinea, Nicaragua. El enfoque de la investigación fue cuantitativo, tipo descriptiva, se tomó una muestra de 100 ganaderos. La información fue recolectada a través de encuestas, y por las características de algunas variables también se hizo uso de formatos de recolección y medición de datos. El procesamiento de la información se realizó con el paquete estadístico SPSS versión 15,0. Se encontró una diversidad representativa de especies de pastos, tanto de pastoreo continuo como de corte, con una predominancia de retana (Ischaemum ciliare) y Brachiaria brizantha cv. Marandú, estos en su mayoría con un manejo poco tecnificado, pues los productores (mujeres y hombres) aún no son conscientes que las áreas de pasturas son un cultivo y deben manejarse como tal, el período de ocupación de las pasturas promedia 7 días, con una carga animal de 2,5 cabezas de ganado/manzana y un periodo de descanso de 23 días, con niveles de degradación que van desde leve hasta severo.

Palabras clave: Manejo de pasturas; pastoreo continuo; período de ocupación; días de descanso; carga animal.

\section{Abstract}

This research was aimed at the characterization of pasture management made by cattle farmers in Nueva Guinea, Nicaragua. The research approach was quantitative, descriptive type, a sample of 100 farmers was taken. The information was collected through surveys, and the characteristics of some variables. Data collection and measurement formats were also used. The information processing was performed with the statistical package SPSS version 15.0. A representative diversity of pasture species was found, both continuous pasture and cut, with a predominance of retana (Ischaemum ciliare) and Brachiaria brizantha cv. (Women and men) are still not aware that pasture areas are crop and should be managed as such, the period of occupation

1 Ingeniero Agroforestal, Profesor de la Universidad de las Regiones Autónomas de la Costa Caribe Nicaragüense, Recinto Universitario Nueva Guinea. Correo: caxy123@yahoo.com

2 Médico Veterinario, Profesor de la Universidad de las Regiones Autónomas de la Costa Caribe Nicaragüense, Recinto Universitario Nueva Guinea. Correo: wcruzpastora@yahoo.com 
of the pastures averages 7 days, with an animal load of 2.5 livestock per block and a rest period of 23 days, with levels of degradation ranging from mild to severe.

Keywords: Pasture management; Continuous grazing; Period of occupation; days off; Animal load.

\section{Introducción}

Nueva Guinea es un municipio de reciente formación y en la actualidad se ha convertido en una de las zonas de mayor potencial ganadero de la región, los suelos del municipio son de vocación forestal; sin embargo, para el 2001, el 6o por ciento del municipio estaba ocupado por pasturas (Instituto Nicaragüense de Estadísticas y Censo (INEC, 2002) y con tendencia a seguir incrementándose esa cifra. Actualmente, las pasturas ocupan un porcentaje del 77 de los suelos de uso agrícola de Nueva Guinea (Álvarez, 2016), ello indica que la actividad ganadera se ha desarrollado progresivamente en la región, se ha diversificado la producción de pasturas tanto en especies como en manejo. Es una realidad, que la desnutrición en bovinos es el factor que más limita la producción del sector pecuario en los países tropicales, el bajo consumo de energía y proteínas disminuye de forma significativa la capacidad productiva de los rumiantes (Vargas y Fonseca, 1989).

Por lo anterior, se consideran importantes los tipos de pastos y su manejo en los sistemas de producción pecuario, ya sean estos especializados (carne, leche) o de doble propósito, no se debe obviar también los efectos negativos de la mala alimentación sobre la reproducción del hato, además de la predisposición a ser afectados por enfermedades, es decir la nutrición además de favorecer la producción, favorece la reproducción y la resistencia a enfermedades en los bovinos. La presente investigación aborda el manejo de los pastizales en los sistemas de producción ganaderos de Nueva Guinea, las especies predominantes y el nivel de degradación que estos presentan.

\section{Revisión de literatura}

\section{La ganadería en el mundo}

Según la Organización de las Naciones Unidas para la Agricultura y la Alimentación (FAO, 2009) el ganado representa el 40\% del valor mundial de la producción agrícola y es la base de los medios de subsistencia y la seguridad alimentaria de casi mil millones de personas.

El sector pecuario, al igual que una gran parte de la agricultura, desempeña una compleja función económica, social y ambiental. La sociedad espera que el sector siga satisfaciendo la creciente demanda mundial de productos animales de modo barato, rápido e inocuo. Debe hacerlo de manera sostenible respecto al medio ambiente, a la 
vez que se controlan la incidencia y las consecuencias de las enfermedades animales y se generan oportunidades de desarrollo rural, reducción de la pobreza y seguridad alimentaria (FAO, 2009).

En cuanto a la producción pecuaria, América Latina y el Caribe fue la segunda Región que más creció entre 1980 y 2007 (después de Asia), especialmente en el caso de la carne (pasó de 15,7 a 40,3 millones de toneladas) y de la leche (pasó de 35 a 68.7 millones de toneladas). Se destaca el dato de Brasil en el caso de la carne, cuya producción casi se multiplicó por cuatro y actualmente representa el 7 por cietno del total mundial (FAO, 2010).

\section{La ganadería en Nicaragua}

Los datos del IV Censo Nacional Agropecuario (2012) indican que la población de ganado bovino de Nicaragua es de 4.13 millones, concentradas en 262,527 fincas agropecuarias. La producción de carne y leche se realiza bajo la modalidad de doble propósito, se estima que el 90 por ciento de la producción de leche proviene de hatos de doble propósito, existiendo especialización en leche en porcentajes no representativos.

Los porcentajes del hato bovino, el El 26,77 lo posee la RACCS; 12,51 Chontales, 10 Matagalpa y el 8,42 Boaco, siendo en estos departamentos donde se producen los mayores volúmenes de producción láctea (Ministerio de Fomento, Industria y Comercio (MIFIC, 2012). En Nueva Guinea se reportan 5,895 productores, de estos el 84 por ciento son ganaderos (Álvarez y Cruz, 2011). El INIDE y MAGFOR en el IV CENAGRO (2012) reportan para la RACCS 266, o58 vacas en producción, de estas, el 31 por ciento $(81,900)$ están en Nueva Guinea. Lo que indica que las actividades relacionadas con la producción láctea tienen un peso importante en la economía de Nueva Guinea.

\section{Sistemas de pastoreo}

Los sistemas de pastoreo ocupan un porcentaje del 26 de la superficie sin hielo terrestre (Steinfeld et al., 2006 citado por FAO, 2009). Los sistemas de pastoreo extensivo cubren la mayoría de las zonas áridas marginales del mundo en lo que respecta a la producción de cultivos.

\section{Manejo de pasturas}

En el trópico los niveles de productividad animal (carne, leche) son inferiores a los obtenidos en pasturas de zonas templadas. Esto se debe en gran medida a que la estructura de la pastura tropical ofrece una densidad menor de hojas verdes que afecta la eficiencia de cosecha por parte del animal ocasionando un menor consumo de proteína y energía digestible, las altas temperaturas y la humedad ambiental que obligan a restringir el consumo durante las horas más calientes del día y aumentar 
el pastoreo nocturno, adicionalmente, en muchas explotaciones por razones de seguridad el ganado es recogido en los corrales durante las noches, generalmente con insuficiente forraje disponible para compensar el menor tiempo de pastoreo diurno, estos factores disminuyen la eficiencia del pastoreo (Faría, 1998).

El uso de gramíneas forrajeras adaptadas a las condiciones ambientales de la finca, bajo una forma organizada de pastoreo en cultivo puro o en combinación con leguminosas forrajeras, la siembra de cultivos forrajeros de altos rendimientos y la incorporación de árboles multipropósitos son algunas de las estrategias que además del riego y las prácticas tradicionales de conservación de forraje (henificación y ensilaje) han permitido mejorar la producción y productividad forrajera a lo largo del año en los trópicos. Para lograr niveles elevados y estables de productividad en la ganadería de doble propósito es necesario un manejo racional del suelo, pasto y animal entre otras cosas evitando el sobrepastoreo, ajustando la carga animal, adecuando los sistemas de pastoreo e incorporando nutrientes al suelo (Faría, 1998).

El aporte del sistema de doble propósito en la producción de leche en el trópico americano supera el 50\%. La ganadería de doble propósito se caracteriza por su sencillez, estabilidad, flexibilidad y liquidez diaria, lo cual le ha permitido sobrevivir, aun bajo situaciones climáticas, económicas y sociales difíciles. La alimentación del ganado está basada principalmente en el pastoreo, dependiendo poco de insumos producidos fuera de la finca. No obstante, en la mayoría de las áreas ganaderas la baja productividad del sistema está asociada a la baja cantidad y pobre calidad del forraje disponible, el potencial genético de los animales y el manejo (Soto, 2005).

\section{Estrategias de manejo y utilización de leguminosas forrajeras}

La desnutrición en bovinos es el factor que más limita la producción en este sector en los países tropicales, el bajo consumo de energía y proteínas disminuye de forma significativa la capacidad productiva de los rumiantes (Vargas y Fonseca, 1989). Por ello es de trascendental importancia los tipos de pastos y el manejo de estos en los sistemas de producción pecuario, ya sean estos especializados o de doble propósito, sin obviar los efectos negativos de la mala alimentación sobre la reproducción del hato, además de la predisposición a ser afectados por enfermedades.

Las estrategias de alimentación y pastoreo con leguminosas tropicales normalmente están basada en que las leguminosas constituyen parte de la dieta por lo que se manejan con el objetivo de aprovechar sus atributos nutritivos para superar o aminorar las limitaciones (ejemplo: bajo contenido de proteína bruta, pobre digestibilidad, elevado tiempo de retención) de la gramínea u otro componente no leguminoso de la ración (Lascano, 2000). 
Una estrategia de manejo flexible de pasturas fue propuesta por Spain et al. (1985). En ella plantea el ajuste de la carga y la frecuencia de pastoreo dependiendo de dos parámetros del pastizal: a) La carga se ajusta cuando la presión de pastoreo alcanza el límite prefijado; b) La frecuencia de pastoreo se ajusta cuando la proporción de leguminosas alcanza el límite seleccionado. No obstante, la estrategia de pastoreo o alimentación puede variar desde sistemas simples a sistemas altamente complejos.

\section{La organización del pastoreo}

Para lograr niveles elevados y estables de productividad en la ganadería de doble propósito es necesario un manejo racional del suelo, pasto y animal entre otras cosas evitando el sobrepastoreo, ajustando la carga animal, adecuando los sistemas de pastoreo e incorporando nutrientes al suelo (Holmann et al. 2004). Lo más recomendable es organizar los animales en grupos con requerimientos alimenticios y de manejos similares y destinar para ellos una superficie de pastoreo (módulos) que será conducida de acuerdo a normas que tengan en cuenta las necesidades de la especie forrajera y la del animal a fin de lograr tener la máxima productividad animal sin afectar el recurso pastizal (Holmann et al, 2004).

\section{El proceso de degradación de pasturas}

La degradación de la tierra es generalmente definida como la reducción temporal o permanente en la capacidad productiva en un agro-ecosistema determinado (Holmann et al, 2004). Latinoamérica es la región del mundo en desarrollo que tiene las mayores áreas degradadas. En el caso de las pasturas, este proceso de degradación está ligado a: (1) establecimiento de pasturas en tierras frágiles (laderas); (2) siembra de pasturas pobremente adaptadas; (3) sobre-pastoreo durante la época lluviosa; (4) quema incontrolada y frecuente de pasturas; y (5) agotamiento de nutrientes (Spain y Gualdrón, 1991). La degradación de las pasturas trae serias consecuencias al productor: reduce primero los rendimientos en producción animal y luego incrementa los costos.

Una vez establecida la pastura mejorada, la deficiencia de nitrógeno es el primer factor que desestabiliza la pastura y provoca el inicio de la degradación (Barcellos, 1986). Una vez declarada la deficiencia del nitrógeno, la calidad y el vigor de la pastura comienzan a declinar e inducen una aguda disminución de la actividad biológica; entonces, otros nutrientes, como el fósforo y el azufre, pueden aparecer como deficientes. Cuando la pastura comienza a perder vigor la invasión de malezas aparece, aumentando aún más el problema.

Después de un período prolongado de utilización de las pasturas, es posible que ocurran cambios importantes en la estructura física del suelo, como la compactación, lo cual aumenta la escorrentía, disminuye el desarrollo de las raíces y la extracción de nutrientes que se encuentran a mayor profundidad en el suelo. Asimismo, la 
compactación del suelo permite que el agua corra por la superficie arrastrando partículas y materiales en depósito, iniciando entonces el proceso de erosión, con lo cual la pastura entra en un proceso de degradación severa. La mayor parte de los 13,2 millones de hectáreas cubiertas de pastos en América Central son de origen antropogénico; pero de estas, entre 50 y $80 \%$ se encuentran degradadas (Holmann et al. 2004).

\section{Metodología para determinar los niveles de degradación de las pasturas}

Tabla 1. Descripción cualitativa y cuantitativa de cada uno de los cuatro niveles de degradación de pasturas

\begin{tabular}{|c|c|c|c|c|}
\hline \multirow{2}{*}{ Síntoma } & \multicolumn{4}{|c|}{ Nivel de degradación } \\
\hline & $1=$ No aparente & $2=$ Leve & 3= Moderado & 4= Severo \\
\hline Color & Verde oscuro & Verde claro & Verde-amarillo & Amarillo \\
\hline Materia muerta & $<10 \%$ & $11-20 \%$ & $21-30 \%$ & $>30 \%$ \\
\hline Suelo desnudo & $<10 \%$ & $11-20 \%$ & $21-30 \%$ & $>30 \%$ \\
\hline Malezas & $<10 \%$ & $\begin{array}{l}\text { 11-20 \% (apari- } \\
\text { ción de malezas } \\
\text { de hoja angos- } \\
\text { ta) }\end{array}$ & $\begin{array}{l}\text { 21-30 \% } \\
\text { (aparición de } \\
\text { malezas de hoja } \\
\text { ancha) }\end{array}$ & $\begin{array}{l}\text { >30 \% (mas } \\
\text { colonización } \\
\text { de la pastura } \\
\text { por gramíneas } \\
\text { nativas) }\end{array}$ \\
\hline Edad & $\begin{array}{l}\text { 1-3 años de esta- } \\
\text { blecido }\end{array}$ & $\begin{array}{l}\text { 4-6 años de } \\
\text { establecido }\end{array}$ & $\begin{array}{l}\text { 7-9 años de esta- } \\
\text { blecido }\end{array}$ & $\begin{array}{l}\text { > } 10 \text { años de } \\
\text { establecido }\end{array}$ \\
\hline
\end{tabular}

\section{Carga animal}

La carga animal se define como el número de animales de cierta categoría que pastorean por unidad de superficie en un tiempo determinado. Es decir, la carga animal es el número de "Unidades Animales" por hectárea en un tiempo determinado. Por ejemplo, 30 vacas de $450 \mathrm{~kg}$ en promedio, en un potrero de 20 hectáreas, equivale a una carga animal de $1.5 \mathrm{UA} / \mathrm{ha}$, en el momento de la observación, porque hay $30 \mathrm{UA}$ en 20 hectáreas, y 30/20 = 1.5 (García y López, 2008).

\section{Ajuste de la carga animal}

La producción de forrajes varía de una época a otra durante el año y de un año a otro, por lo que la carga animal se debe ajustar para permitir que exista suficiente forraje disponible aún en las épocas desfavorables, minimizar las pérdidas del forraje producido y evitar el agotamiento del potrero (Engormix, 2010). El productor controla la presión de pastoreo a través de la regulación del número y tipo de animales por hectárea (carga animal) y determina a través del método de pastoreo, la ubicación espacial y temporal de los animales en los diferentes potreros (Engormix, 2010). 
La intensidad del pastoreo afecta tanto la producción de animal como la producción y composición del pastizal. A baja presión de pastoreo, la producción por hectárea es baja ya que el sub-pastoreo trae como consecuencia una pobre utilización de la pastura. Con incrementos en la presión de pastoreo se alcanzan mejoras en la producción por hectárea, aunque decline la productividad por animal. La máxima producción se obtiene cuando la pérdida en producción por animal se compensa con el mayor número de animales por hectárea. Cuando la presión de pastoreo es demasiado alta se disminuye la producción por animal, se deteriora el potrero y se puede incluso presentar erosión del suelo (Engormix, 2010).

Tanto el sobrepastoreo como el sub-pastoreo son perjudiciales para la pastura. Lograr una presión de pastoreo óptima, donde la pastura muestre una buena producción de forraje con la composición botánica deseada y a la vez se obtengan excelentes producciones de carne y/o leche es sumamente complicado, especialmente en el medio tropical. En los sistemas pastoriles es prácticamente imposible conjugar simultáneamente una máxima intersección de energía solar, con tasas máximas de crecimiento del pasto, altos porcentajes de cosecha y alta eficiencia de conversión del forraje consumido (Parson y Penning, 1988).

\section{Capacidad de carga}

Los términos "carga animal" y "capacidad de carga" tienen significados diferentes. Carga animal, es el número de animales por hectárea de pasto por un período definido de tiempo, mientras capacidad de carga se refiere a la capacidad de producción de forraje (García y López, 2008). La capacidad de carga depende de la especie de forraje, la productividad del suelo, el sistema de manejo que se tenga, y el clima, en particular, la cantidad de lluvia y sol. La capacidad de carga se mejora rehabilitando periódicamente las praderas, e incluyendo suficiente sombra y agua disponible para los animales. Por eso es muy importante que las pasturas se mantengan en buenas condiciones, de lo contrario, paulatina, pero inexorablemente, se perderá capacidad de carga (García y López, 2008).

\section{Periodo de ocupación}

Es el tiempo o número de días que un lote de ganado ocupa un potrero del total de los empleados en la rotación. Debe ser lo suficientemente corto para que el rebrote de las plantas que fueron pastoreadas a inicios del período de ocupación no vaya a ser desfoliado por los animales en el mismo ciclo de pastoreo (García y López, 2008). 


\section{Período de descanso}

Es el número de días que permanece el potrero sin pastorear, desde el momento que los animales salen del potrero hasta su regreso. Debe ser lo suficientemente largo para que las plantas superen la mayor tasa de crecimiento diario y almacenen en sus raíces y órganos especializados suficientes reservas para asegurar un rebrote vigoroso luego de la defoliación. Por otra parte, debe ser lo suficientemente corto para evitar la sobremaduración del pasto y una caída drástica del valor nutritivo, especialmente en las gramíneas. La duración más adecuada del período de descanso dependerá de la finca, de la especie, nivel de fertilización, época del año, entre otras (García y López, 2008).

\section{Materiales y métodos}

La investigación se desarrolló en 100 fincas ganaderas del municipio de Nueva Guinea, abarcó 20 comunidades, se condujo bajo el enfoque cuantitativo, de tipo no experimental descriptiva, de corte transversal. Para la selección de las y los productores se consideró que tuvieran ganado bovino, áreas de pastos y disponibilidad de colaborar en el estudio. La recolección de la información se hizo a través de encuestas, y por las características de algunas variables también se usaron formatos de recolección y medición de datos. El procesamiento de la información se realizó con el paquete estadístico SPSS versión 15.0.

Para la evaluación de los niveles de degradación de las áreas de pastos se utilizó la metodología propuesta por Barcellos, 1986 y las imágenes descriptivas de Holmann et $a l$, 2004, y para la evaluación del sistema de ganadero en general se coordinó con los productores previamente a la visita para que estos nos acompañaran en el recorrido por cada sistema ganadero.

\section{Resultados y discusión}

\section{Características generales de los productores y productoras participantes en el estudio}

La tipología de productores en los sistemas ganaderos determina en cierta medida o al menos influenciará en las características de estos, en Nueva Guinea, la mayoría de los productores son pequeños ganaderos (figura 2), estos manejan sistemas de producción que van desde 8 hasta 50 manzanas; sin embargo, no se encontró diferencia significativa entre los sistemas de producción por tipología. 


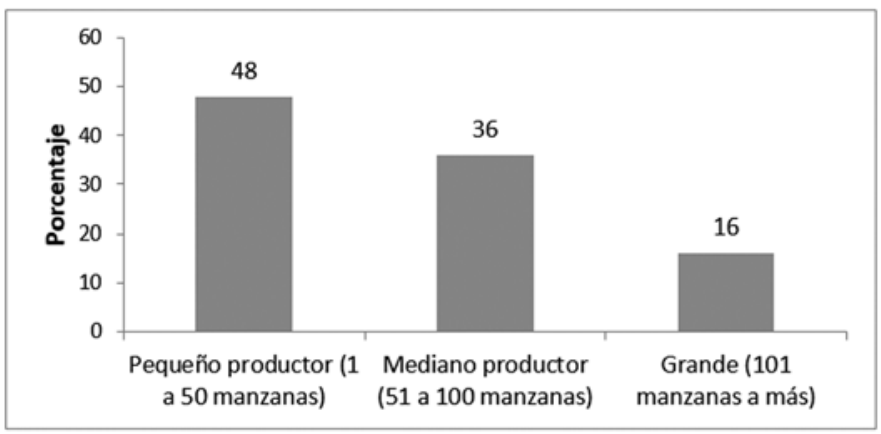

Figura 2: Tipología de productores

Tabla 2: Estadísticos para el tamaño de finca relacionado a la tipología de productores

\begin{tabular}{|c|c|c|}
\hline \multicolumn{2}{|c|}{ Media (mz/productor) } & 82 \\
\hline \multicolumn{2}{|l|}{ Mediana } & 52 \\
\hline \multicolumn{2}{|l|}{ Moda } & 50 \\
\hline \multicolumn{2}{|c|}{ Desviación típica } & 81 \\
\hline \multicolumn{2}{|l|}{ Mínimo } & 8 \\
\hline \multicolumn{2}{|l|}{ Máximo } & 600 \\
\hline \multirow[t]{3}{*}{ Percentiles } & 25 & 40 \\
\hline & 50 & 52 \\
\hline & 75 & 100 \\
\hline
\end{tabular}

La asistencia técnica juega un papel determinante en la conversión de sistemas ganaderos tradicionales a sistemas eficientes, esta debe significar cambios de actitud y tecnológicos en los patrones de producción sobre todo en sistemas que por tradición han sido extensivos.

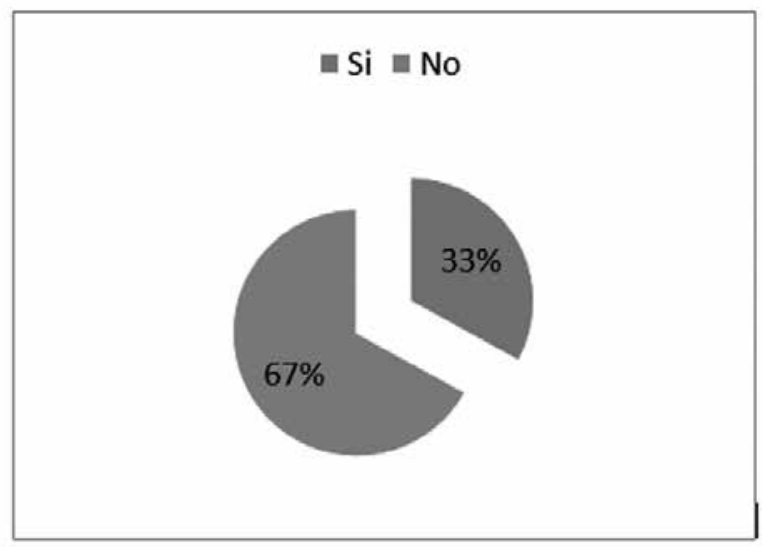

Figura 3: Acceso a la asistencia técnica 
La mayoría de las y los productores participantes en el estudio afirma no recibir asistencia técnica (figura 3), a la vez, la mayoría afirma que ninguna institución les ha visitado ofreciéndole este servicio, las instituciones presentes y oferentes de asistencia técnica son: MAGFOR, INTA y COOPROLECHE, aunque con poca cobertura y en rubros que en ocasiones no tienen que ver con el manejo de pasturas.

La asistencia técnica generalmente es brindada por los programas de gobiernos a través de instancias oficiales, en otros casos por proyectos ejecutados por algunas ONG y en ocasiones una mixtura de ambas, sea cual sea su origen, la asistencia técnica debe garantizarse con calidad, permanencia, pertinencia y amplia cobertura, además debe procurar que esta tenga efectos que se reflejen en los niveles de adopción de los conocimientos puestos a su disposición, esto para que no se genere dependencia absoluta de los productores hacia las agencias de extensión. Generalmente sucede que, al ser la asistencia técnica gratuita, los beneficiaros de esta, no le dan importancia que requiere y cuando esta se deja de brindar, los procesos se detienen porque los beneficiarios no asumen la responsabilidad de adoptar y mantener adecuadamente los procesos en que han sido asistidos.

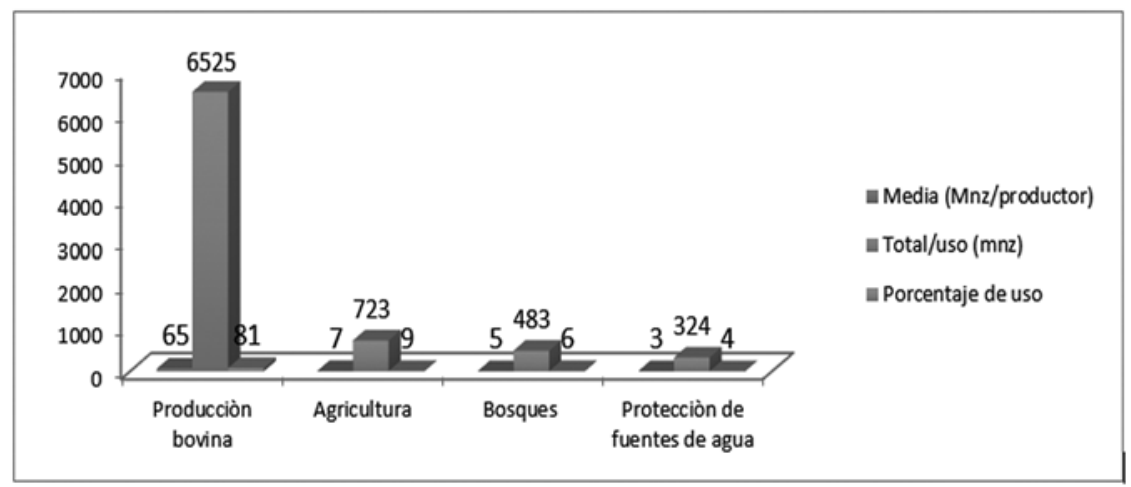

Figura 4: Uso actual del suelo en los sistemas de producción evalaudos

En cuanto al uso actual del suelo (figura 4 y tabla 3 ), es evidente que por las características del municipio la gran mayoría de los productores se dedican a la actividad ganadera, otros ejercen la agricultura y ganadería, el 81\% de los sistemas de producción evaluados se dedican a la actividad ganadera, llama la atención que se está descuidando la protección de fuentes de agua $(3.2 \mathrm{mz} /$ productor) y el manejo de los remanentes de bosques ( $4.8 \mathrm{mz} /$ productor) en las fincas, esto como parte del uso del suelo y como parte de la conservación y sustentabilidad de dichos sistemas, lo que a mediano plazo representa riesgos para los sistemas de producción, pues ellos dependen en gran medida de la adaptación que hagan los ganaderos a situaciones climáticas adversas y de las medidas de mitigación para enfrentar los nuevos escenarios productivos. 
Tabla 3: Estadísticos para los usos del suelo

\begin{tabular}{|c|c|c|c|c|c|}
\hline \multirow{2}{*}{\multicolumn{2}{|c|}{$\begin{array}{l}\text { Muestra (unidades de } \\
\text { producción) }\end{array}$}} & $\begin{array}{l}\text { Producción } \\
\text { bovina }\end{array}$ & Agricultura & Bosques & $\begin{array}{c}\text { Protección } \\
\text { de fuentes } \\
\text { de agua }\end{array}$ \\
\hline & & 100 & 100 & 100 & 100 \\
\hline \multicolumn{2}{|l|}{ Media (mz) } & 65.3 & 7.2 & 4.8 & 3.2 \\
\hline \multicolumn{2}{|c|}{ Mediana (mz) } & 44.0 & 4.0 & 1.0 & 1.0 \\
\hline \multicolumn{2}{|l|}{ Moda (mz) } & 50.0 & 0.0 & 0.0 & 0.0 \\
\hline \multicolumn{2}{|c|}{ Desviación típica (mz) } & 72.5 & 11.1 & 8.7 & 7.6 \\
\hline \multicolumn{2}{|l|}{ Mínimo (mz) } & 5.0 & 0.0 & 0.0 & 0.0 \\
\hline \multicolumn{2}{|c|}{ Máximo (mz) } & 560.0 & 70.0 & 40.0 & 60.0 \\
\hline \multicolumn{2}{|l|}{ Suma (mz) } & 6525.0 & 722.5 & 482.5 & 323.5 \\
\hline \multirow[t]{3}{*}{ Percentiles } & 25 & 26.3 & 1.0 & 0.0 & 0.0 \\
\hline & 50 & 44.0 & 4.0 & 1.0 & 1.0 \\
\hline & 75 & 74.8 & 10.0 & 5.0 & 4.0 \\
\hline
\end{tabular}

\section{Características de las áreas de pastos}

Se encontraron 7 especies de pastos (tabla 4 y 5) con mayor predominancia, el pasto retana a pesar de sus limitaciones en periodo seco sigue siendo una de las especies que los productores aun manejan, el avance en mejora de pasturas es lento, pero se ha dado. Otro elemento a destacar es que las y los productores, a pesar de ser necesario, no han incursionado en el establecimiento de pastos de corte, solo se encuentran pequeñas áreas, este tipo de pastos son el complemento adecuado para la suplementación del ganado en épocas secas y en categorías de alta exigencia nutricional como las vacas en producción.

La nutrición tiene relación directa con la expresión productiva del hato, sea cual sea la categoría en manejo, los ingresos de los productores serán mayores por mayor disposición de producción, se debe tener en cuenta que las condiciones edáficas del municipio no permiten que los pastos suplan los requerimientos nutricionales del ganado, sobre todo a vacas en producción y gestantes, ganado para carne y en periodos de sequía. Muchos productores consideran que la suplementación nutricional es un gasto sin retornos, esta concepción ha de cambiar, pues la adecuada nutrición al hato se traduce en mayores utilidades a los productores. 
Tabla 4. Especies de pasto predominantes

\begin{tabular}{|c|c|c|c|c|c|c|c|c|}
\hline \multirow{2}{*}{\multicolumn{2}{|c|}{$\begin{array}{l}\text { Muestra (unidades de } \\
\text { producción) }\end{array}$}} & $\begin{array}{l}\text { Retana } \\
\text { (Ischaemum } \\
\text { ciliare) }\end{array}$ & $\begin{array}{c}\text { Brachiaria } \\
\text { brizantha cv. } \\
\text { Marandú }\end{array}$ & $\begin{array}{c}\text { Taiwán } \\
\text { (Pennisetum } \\
\text { purpureum) }\end{array}$ & $\begin{array}{l}\text { Brachiaria } \\
\text { brizantha cv. } \\
\text { Toledo }\end{array}$ & $\begin{array}{l}\text { Jaragua } \\
\text { (Hyparrhenia } \\
\text { rufa) }\end{array}$ & $\begin{array}{c}\text { India } \\
\text { (Panicum } \\
\text { maximum) }\end{array}$ & $\begin{array}{c}\text { Panicum } \\
\text { maximum } \\
\text { cv. Mombaza }\end{array}$ \\
\hline & & 100 & 100 & 100 & 100 & 100 & 100 & 100 \\
\hline \multicolumn{2}{|l|}{ Media (mz) } & 38.54 & 16.68 & 1.59 & 1.95 & 0.64 & 0.44 & 1.13 \\
\hline \multicolumn{2}{|c|}{ Mediana (mz) } & 26.5 & 9.5 & O & 0 & O & O & 0 \\
\hline \multicolumn{2}{|l|}{ Moda (mz) } & 20 & $\mathrm{O}$ & $\mathrm{O}$ & 0 & 0 & 0 & $\mathrm{O}$ \\
\hline \multicolumn{2}{|c|}{ Desviación típica (mz) } & 53 & 23 & 3 & 6 & 4 & 2 & 4 \\
\hline \multicolumn{2}{|c|}{ Mínimo (mz) } & 0 & $\mathrm{O}$ & $\mathrm{O}$ & $\mathrm{O}$ & 0 & $\mathrm{O}$ & $\mathrm{O}$ \\
\hline \multicolumn{2}{|c|}{ Máximo (mz) } & 470 & 140 & 20 & 35 & 32 & 20 & 30 \\
\hline \multicolumn{2}{|l|}{ Suma (mz) } & 3854.5 & 1668.5 & 159.75 & 195.5 & 64 & 44 & 113 \\
\hline \multirow[t]{3}{*}{ Percentiles } & 25 & 15 & 2 & $\mathrm{O}$ & $\mathrm{O}$ & $\mathrm{O}$ & $\mathrm{O}$ & $\mathrm{o}$ \\
\hline & 50 & 26.5 & 9.5 & 0 & 0 & 0 & O & 0 \\
\hline & 75 & 45 & 20 & 2.75 & 0 & 0 & 0 & 0 \\
\hline
\end{tabular}

Entre las limitantes del pasto retana destacan: forma una masa (colchón) en meses de mayor precipitación, material maduro y poco palatable, sistema radicular muy superficial que no le permite extraer nutrientes ni humedad de las capas inferiores del suelo, la cantidad de proteína bruta es baja, oscila entre 6 y 7 por ciento. Sin embargo, los pastos del género Panicum son recomendables porque tienen resistencia a la sequía, sombra, quema y pisoteo, el contenido de proteína bruta oscila entre 10 a 14 por ciento (INATEC, 2016).

Tabla 5: Tamaño de potreros en relación a la especie de pasto

\begin{tabular}{|l|l|l|}
\hline \multicolumn{1}{|c|}{ Especie } & \multicolumn{1}{|c|}{$\begin{array}{c}\text { Tamaño de } \\
\text { potreros (mz) }\end{array}$} & Total (mz/especie) \\
\hline Retana (Ischaemum ciliare) & 38 & 3854 \\
\hline Brachiaria brizantha cv. Marandú & 17 & 1668 \\
\hline Taiwán (Pennisetum purpureum) & 1.6 & 160 \\
\hline Brachiaria brizantha cv. Toledo & 2 & 195 \\
\hline Jaragua (Hyparrhenia rufa) & 0.64 & 64 \\
\hline India (Panicum maximum) & 0.44 & 44 \\
\hline Panicum maximum cv. Mombaza & 1.13 & 113 \\
\hline
\end{tabular}




\section{Niveles de degradación de las áreas de pastos}

Entre la diversidad de pastos encontrados, la retana (Ischaemum ciliare) es predominante, sin embargo, el Brachiaria brizantha presenta los mayores niveles de degradación severa (77\%), (tabla 6 y figura 5)

En las pasturas, el proceso de degradación está ligado a: (1) establecimiento de pasturas en tierras frágiles; (2) siembra de pasturas pobremente adaptadas; (3) sobrepastoreo durante la época lluviosa; (4) quema incontrolada y frecuente de pasturas; y (5) agotamiento de nutrientes (Spain y Gualdrón, 1991). La degradación de pasturas trae serias consecuencias al productor: reduce primero los rendimientos en producción animal y luego incrementa los costos. Los factores que están determinando los niveles de degradación en las pasturas de Nueva Guinea están asociados al sobrepastoreo, largos periodos de ocupación de los potreros, reducido tiempo de reposo, baja fertilidad del suelo, poca incorporación de materia orgánica (estiércol) a las pasturas, inadecuada planificación del pastoreo y tamaño inadecuados de potreros (potreros muy grandes o muy pequeños) que permitan su uso óptimo.

Tabla 6: Niveles de degradación de pasturas por especie

\begin{tabular}{|l|c|c|c|c|c|c|c|}
\hline Nivel de degradación (\%) & Retana & B. Brizantha & Taiwán & Toledo & Jaragua & India & Mombaza \\
\hline No aparente & 0 & 1 & 7 & 0 & 0 & 0 & 0 \\
\hline Leve & 15 & 2 & 19 & 16 & 2 & 2 & 12 \\
\hline Moderado & 64 & 48 & 7 & 2 & 1 & 2 & 1 \\
\hline Severo & 17 & 77 & 1 & 1 & 0 & 0 & 0 \\
\hline No tiene esa especie & 4 & 78 & 66 & 81 & 97 & 96 & 97 \\
\hline Total & $\mathbf{1 0 0}$ & $\mathbf{1 0 0}$ & $\mathbf{1 0 0}$ & $\mathbf{1 0 0}$ & $\mathbf{1 0 0}$ & $\mathbf{1 0 0}$ & $\mathbf{1 1 0}$ \\
\hline
\end{tabular}

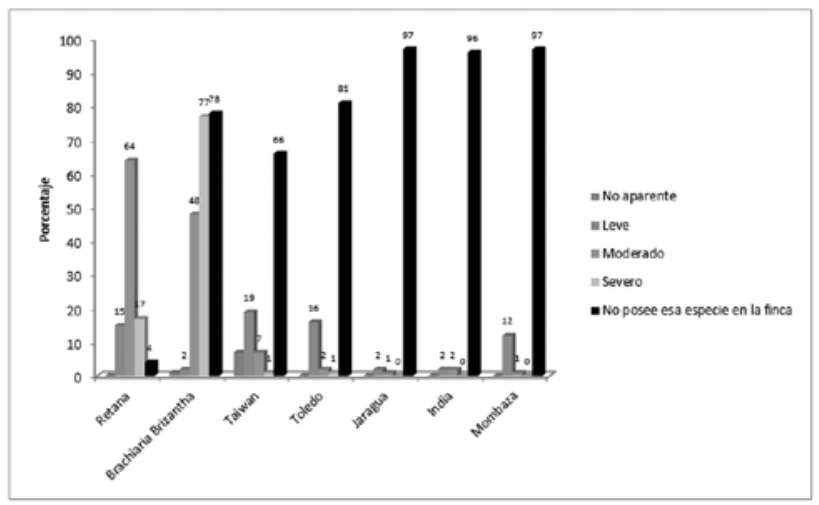

Figura 5: Niveles de degradación de las especies predominantes de pasto 


\section{Manejo de las pasturas}

La carga animal (figura 6) en las unidades de producción en estudio es de 2.5 cabezas de ganado $/ \mathrm{mz}$, con rangos que oscilan entre 1 y 14 cabezas de ganado $/ \mathrm{mz}$, con una ocupación de 7 días por potrero y 23 días de descanso. Lo deseable sería $1 \mathrm{UA} / \mathrm{mnz}$ en verano y 2 en invierno (García y López, 2008).

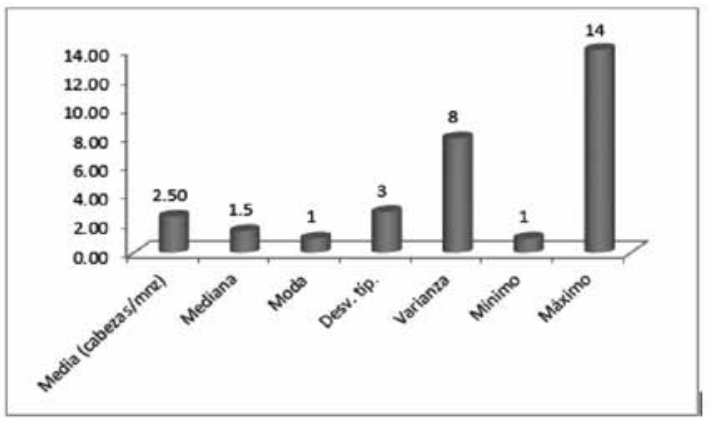

Figura 6: Estadísticos para la carga animal (cabezas/mz)ensistemasdepastoreodeNuevaGuinea.

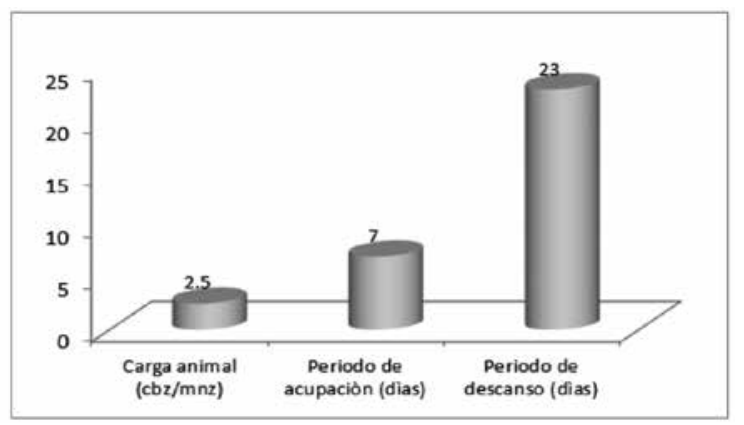

Figura 7: Carga animal y período de ocupación en áreas de pasturas de Nueva Guinea.

Los ganaderos deben tener en cuenta que el periodo de descanso de los potreros debe ser lo suficientemente largo para que las plantas superen la mayor tasa de crecimiento diario y almacenen en sus raíces y órganos especializados las reservas necesarias para asegurar un rebrote vigoroso luego de la defoliación, a la vez evitar la sobremaduración del pasto y con ello reducción del valor nutricional.

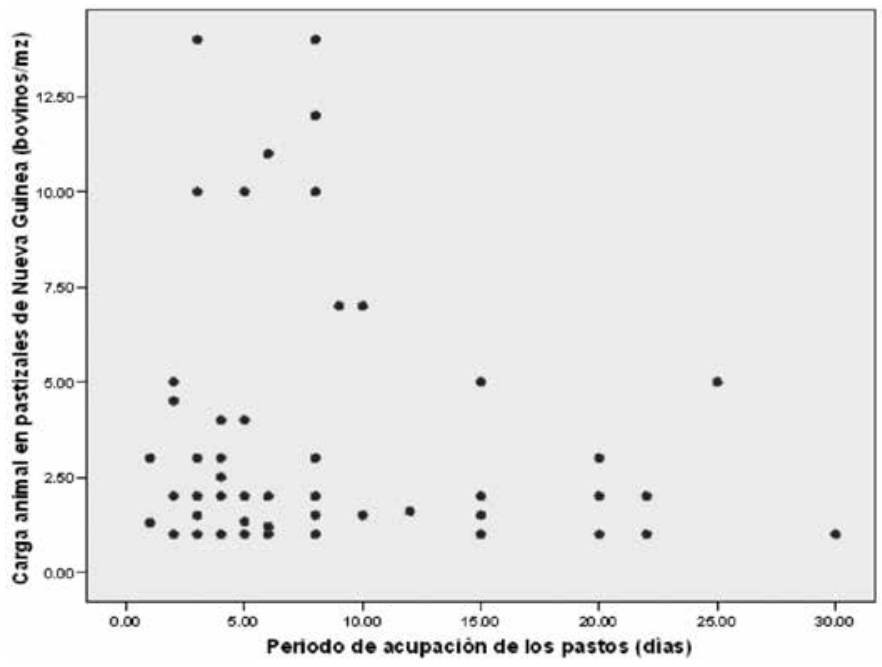

Figura 8: Distribución de la carga animal y los períodos de ocupación en sistemas ganaderos de Nueva Guinea. 
Los ganaderos, en su mayoría controlan las malezas de los pastizales de forma manual cada 6 meses (gráfico 9 y 10), sin importar el método de control de malezas, este aspecto es determinante para garantizar el rendimiento y calidad óptima de las pasturas.

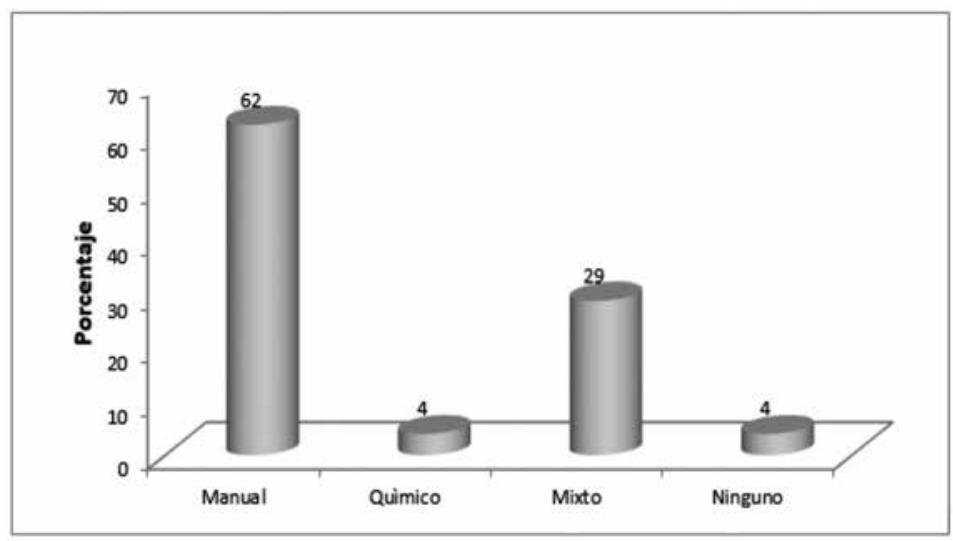

Figura 9: Formas de control de malezas áreas de pasturas de Nueva Guinea.

INATEC (2016) plantea que es necesario eliminar las malezas de las pasturas, pues estas absorben los nutrientes disponibles para los pastos, bloquean el sol y reducen la productividad de los mismos. El control de malezas es relevante sobre todo durante los 3 primeros meses después de la siembra.

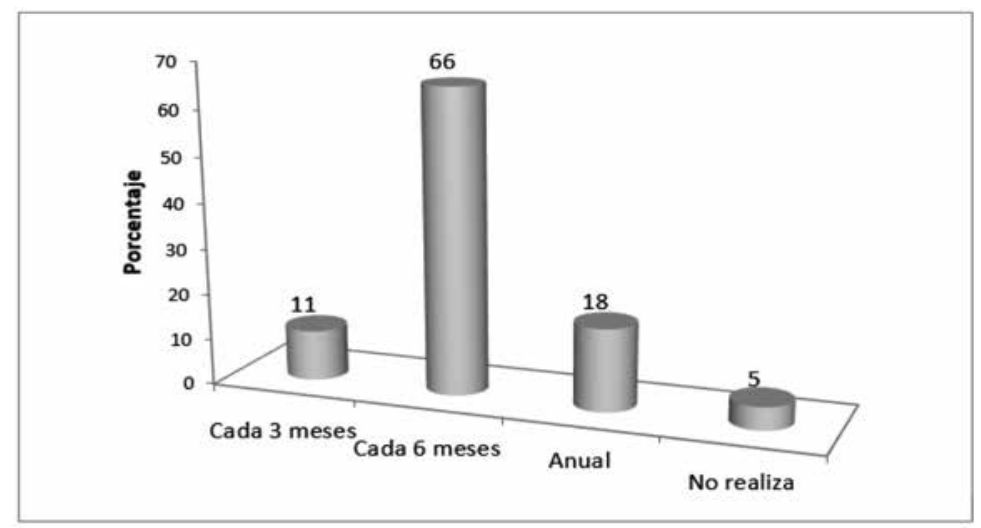

Figura 10: Frecuencias con la que se hace el control de malezas en áreas de pasturas de Nueva Guinea.

Entre las causas que pueden propiciar la invasión de malezas en los pastizales se destacan: el mal manejo de los pastos, mala preparación del terreno, uso de la semilla infectada por malezas, traslado de ganado de un potrero infectado a otro limpio, el consumo de las semillas de malezas ingeridas durante el pastoreo y suplementación, luego son excretadas en las heces. Como resultado de la invasión de malezas en los pastizales se obtiene: competencia por nutrientes, agua, luz y espacio, lo que a su vez 
genera es el bajo crecimiento de los pastos, un bajo valor nutritivo, la aparición de plagas y enfermedades y el aumento de los costos de producción. Cuando el ganado reduce la cantidad de consumo nutricional, no se puede obtener la productividad esperada (INATEC, 2016).

En lo referido a la fertilización como parte del manejo de las pasturas es deficiente (figura 11), no existe un programa regular de fertilización y en los suelos de estas zonas esto puede ser determinante para evitar la degradación de las pasturas a niveles severos.

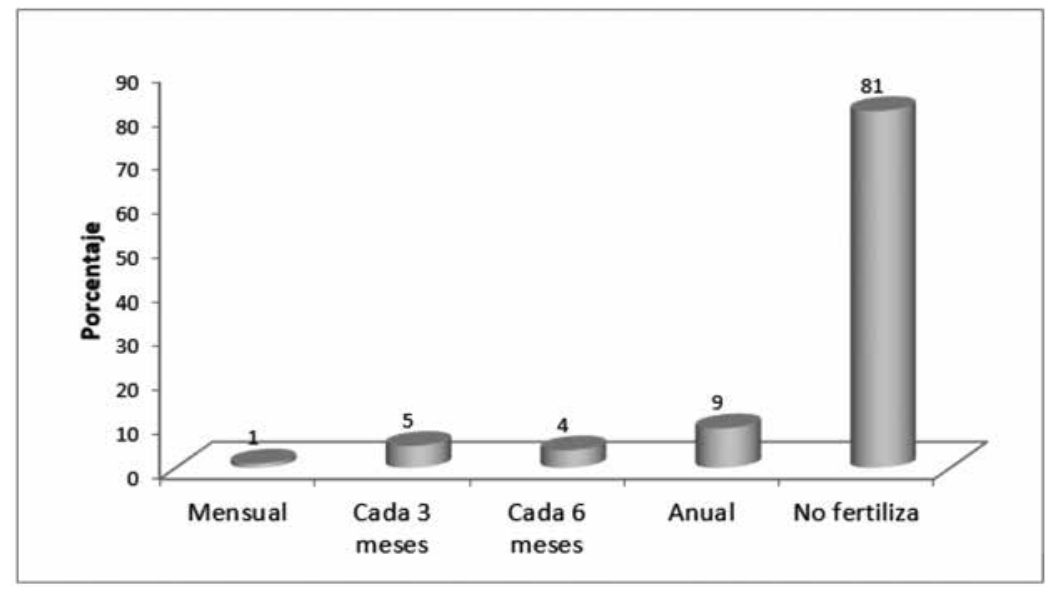

Figura 11: Fertilización en áreas de pastos de Nueva Guinea.

Cuando se realiza la fertilización antes de la siembra, las malezas absorben los fertilizantes, por lo que la disponibilidad de nutrientes baja para el cultivo. Por tal razón se debe fertilizar después de 25 días de germinada la semilla. En el caso de los fertilizantes orgánicos se deben aplicar antes de la siembra para que se incorporen los nutrientes al suelo (INATEC, 2016). Los ganaderos, en su mayoría no están haciendo uso del estiércol bovino para integrarlo como fertilizante natural a las pasturas (solamente el $29 \%$ lo hace), si lo hicieran los resultados productivos serían mayores y los ahorros por compra de fertilizantes, en el caso de los que fertilizan serían significativos.

\section{Conclusiones}

Se puede concluir que los pastos en los sistemas ganaderos del municipio presentan niveles de degradación significativos, asociados a la utilización de variedades pobremente adaptadas a las condiciones climáticas, manejo inadecuado del pastoreo, mal manejo de la fertilización y sobrepastoreo que ocasiona bajas en la productividad animal y en consecuencia pérdidas económicas a los ganaderos. La falta de cobertura de asistencia técnica es un factor determinante para un adecuado manejo de las pasturas. 


\section{Lista de referencias}

Álvarez, C. (2016). Modelos de producción y usos del suelo en Nueva Guinea, conferencia en 1er foro sobre sobre medio ambiente, estado del agua y el suelo, fundación Luz en la selva, Nueva Guinea, RACCS. 01 y o2 de agosto de 2016.

Álvarez, C., y Cruz W. (2011). Manejo de pasturas e implementación del componente arbóreo en las áreas de pastos de Nueva Guinea, RACCS. Nicaragua.

Barcellos, A. (1986). Recuperacão de pastagens degradadas. Empresa Brasileira de Pesquisa Agropecuaria (Embrapa-CPAC).

FAO (2009). Estado mundial de la agricultura y la alimentación: La ganadería, a examen. Roma.

FAO (2010). Aumentan consumo y producción de productos pecuarios en América Latina y el Caribe. Disponible en: http://www.fao.org/americas/noticias/ver/ es/c/23069o/. Santiago de Chile, 18 de febrero de 2010

Faría, J. (1998). Manejo de pastos y forrajes en la ganadería de doble propósito, Venezuela.

García, T. y López, I. (2008). Cómo estimar carga animal para pastoreo continuo. Campo Experimental La Posta, Paso del Toro, CIRGOC-INIFAP, México.

Holmann, F. A., Rivas, L., White, D., Estrada, R. D., Burgos, C., Pérez, E. Ramírez, G. y Medina, A. (2004). ¿Vale la pena recuperar pasturas degradadas? Una evaluación de los beneficios y costos desde la perspectiva de los productores y extensionistas pecuarios en Honduras.

INEC (2002). III Censo Nacional Agropecuario (CENAGRO). Nicaragua.

INIDE y MAGFOR (2012). IV censo nacional agropecuario (IV CENAGRO). Nicaragua.

INIDE y MAGFOR. (2011). IV Censo Nacional Agropecuario (CENAGRO). Nicaragua.

INATEC (2016). Pastos y forrajes - Manual del protagonista, proyecto MEFOTEC.

Lascano, C.E. (200o). Selective grazing on grass-legume mixture in tropical pastures. InLemaire, G., Hodgson, J.de, Moraes, A., Nabinger, C. and P. C de F. Carvalho, eds. Grassland Ecophysiology and Grazing Ecology. CAB International. Curitiba, Parana. 
MIFIC (2012). Análisis de encadenamiento productivo para la generación de valor agregado en nueve cadenas agroalimentarias ubicadas en las zonas demayor potencial productivo de Nicaragua. MIFIC, Managua, Managua.

Parson, A. J. y Penning, P.D. (1988). The effect of the duration of regrowth on photosynthesis, leaf death and the average rate of growth in rotationally grazed sward. Grass and Forage Science 43, 15-27.

Soto B. E. (2005). La ganadería de doble propósito en Venezuela. In Memorias del XII Congreso Venezolano Producción e Industria Animal. AVPA, INIA, UCV, Maracay.

Spain, J. M. y Gualdrón, R. (1991). Degradación y rehabilitación de pasturas.

Spain, J., Pereira, J. M. and Gualdron, R. (1985). A flexible grazing management system proposed for the advance evaluation of asociation of tropical grasses and legume. In Proceeding of the International Grassland Congress. The Council of Japan, Nishi Nasuno, Kyoto, Japan.

Vargas, E. y Fonseca, H. (1989). Contenido mineral y proteico de forrajes, editorial de la universidad de Costa Rica.

Vargas, E., Fonseca, H. (1989). Contenido mineral y proteico de forrajes. editorial de la universidad de Costa Rica.

www.engormix.com (s.f.). Cómo estimar carga animal, recuperado en junio de 2010. 